\title{
Erratum to: Factors associated with adherence to Antiretroviral Therapy (ART) among adult people living with HIV and attending their clinical care, Eastern Ethiopia
}

\author{
Shiferaw Letta ${ }^{1 *}$, Asrat Demissie $^{2}$, Lemessa Oljira ${ }^{3}$ and Yadeta Dessie ${ }^{3}$
}

It has come to the publisher's attention that in the original article [1], Table 3 was incorrectly formatted. The correct formatting of the table has been updated in the original article, and published in this Erratum for quick reference, see Table 1.

In addition, the authors noticed errors in certain reference citations within the discussion section following the publication of [1]. The section of text affected has now been amended in the original article [1], and a list of the changes made has been listed for quick reference:

The correct reference for 'Yirgalam' is: Markos E, Worku A, Davey G. Adherence to ART in PLWHA at Yirgalem hospital, South Ethiopia. Ethiop J Health Dev. 2008;22(2):174-9.

The correct references for 'Jimma' are: Amberbir A, Woldemichael K, Getachew S, Girma B, Deribe K. Predictors of adherence to antiretroviral therapy among HIV-infected persons: a prospective study in Southwest Ethiopia. BMC Public Health. 2008;8:265; and Tiyou A, Belachew T, Alemseged F, Biadgilign S. Predictors of adherence to antiretroviral therapy among people living with HIV/AIDS in resource-limited setting of southwest Ethiopia. AIDS Res Ther. 2010;7:39.

The correct references for 'Nigeria' are: Bello SI. HIV/ AIDS patients' adherence to Antiretroviral therapy in Sobi specialist hospital, Ilorin Nigeria. J Adv Sci Res. 2011;2(3):52-7; and Erah P, Arute J. Adherence of HIV/ AIDS patients to antiretroviral therapy in a tertiary health facility in Benin City, Nigeria. Afr J Pharm Pharmacol. 2007;2(7):145-52.
The correct reference for 'Kenya' is: Anthony N. Factors that influence non-adherence to antiretroviral therapy among HIV and AIDs patients in central province, Kenya, 2011. http://ir-library.ku.ac.ke/handle/123456789/1725.

\section{Author details}

${ }^{1}$ Haramaya University, College of Health and Medical Sciences, School of Nursing and Midwifery, Harar, Ethiopia. ${ }^{2}$ Addis Ababa University, School of Nursing and Midwifery, Addis Ababa, Ethiopia. ${ }^{3}$ Haramaya University, College of Health and Medical Sciences, Department of Public Health, Harar, Ethiopia.

Published online: 02 March 2016

\section{Reference}

1. Letta S, Demissie A, Lemessa O, Dessie Y. Factors associated with adherence to Antiretroviral Therapy (ART) among adult people living with HIV and attending their clinical care, Eastern Ethiopia. BMC Int Health Hum Rights. 2015;15:33. doi:10.1186/s12914-015-0071.

* Correspondence: shife1973@gmail.com

${ }^{1}$ Haramaya University, College of Health and Medical Sciences, School of

Nursing and Midwifery, Harar, Ethiopia

Full list of author information is available at the end of the article 
Table 1 Factors associate with ART adherence among adult patients on ART in public health institutions in Harar and Dire Dawa towns, Eastern Ethiopia, 2012

\begin{tabular}{|c|c|c|c|c|}
\hline \multirow[b]{2}{*}{ Characteristics } & \multicolumn{2}{|l|}{ Adherence status } & \multirow[b]{2}{*}{ COR $(95 \% \mathrm{Cl})$} & \multirow[b]{2}{*}{ AOR $(95 \% \mathrm{Cl})$} \\
\hline & Adhered No (\%) & Not Adhered No (\%) & & \\
\hline \multicolumn{5}{|l|}{ Age in years } \\
\hline $18-24$ & $32(78.8)$ & $9(22.0)$ & $0.97(0.42-2.25)$ & $0.84(0.31-2.25)$ \\
\hline $25-34$ & $226(86.3)$ & $36(13.7)$ & $1.71(1.00-2.29)$ & $1.27(0.68-2.37)$ \\
\hline $35-44$ & 159 (89.9) & $18(10.2)$ & $2.41(1.28-4.54)$ & $2.40(1.15-5.01)^{*}$ \\
\hline$>=45$ & $110(78.6)$ & $30(21.4)$ & 1 & 1 \\
\hline \multicolumn{5}{|c|}{ Average income/month } \\
\hline$<500.00 \mathrm{ETB}$ & $123(94.6)$ & $7(5.4)$ & 1 & 1 \\
\hline 501-999.00 ETB & $61(92.4)$ & $5(7.6)$ & $4.09(1.828-9.187)$ & $6.73(2.71-16.75)^{*}$ \\
\hline$>1000.00 \mathrm{ETB}$ & $60(80.0)$ & $15(20.0)$ & $2.85(1.100-7.359)$ & $1.62(0.57-4.58)$ \\
\hline Not determined & $283(81.1)$ & $66(18.9)$ & $0.93(0.99-1.745)$ & $1.21(0.59-2.49)$ \\
\hline \multicolumn{5}{|l|}{ Waiting time } \\
\hline$\leq 30 \min$ & $364(87.5)$ & $52(12.5)$ & $1.70(1.12-2.76)$ & $1.36(0.79-2.34)$ \\
\hline$<30 \min$ & $163(79.9)$ & $41(20.1)$ & 1 & \\
\hline \multicolumn{5}{|l|}{ Depressiom } \\
\hline Yes & $257(81.3)$ & $59(18.7)$ & $0.55(0.35-0.87)$ & $0.36(0.213-0.614)^{*}$ \\
\hline No & $270(88.8)$ & $34(11.2)$ & 1 & 1 \\
\hline \multicolumn{5}{|l|}{ Pill burden } \\
\hline 2 tablets & $221(84.7)$ & $40(15.3)$ & $3.45(1.08-11.09)$ & $12.98(2.781-60.59)^{*}$ \\
\hline 3 tablets & $257(87.7)$ & $36(12.3)$ & $4.46(1.38-14.38)$ & $12.90(2.87-57.94)^{*}$ \\
\hline 4 tablets & $41(77.4)$ & $12(22.6)$ & $2.4(0.59-7.75)$ & $5.87(1.21-28.54)^{*}$ \\
\hline 5 tablets and more & $8(61.5)$ & $5(38.5)$ & 1 & 1 \\
\hline \multicolumn{5}{|l|}{ Substance use } \\
\hline Used & $158(77.8)$ & $45(22.2)$ & $0.46(0.29-0.71)$ & $0.612(0.37-1.03)$ \\
\hline Not-used & $369(88.5)$ & $48(11.5)$ & 1 & \\
\hline \multicolumn{5}{|c|}{ Opportunistic infections(Ols) } \\
\hline Not encountered & $198(89.2)$ & $24(10.8)$ & $1.73(1.05-2.24)$ & $2.81(1.47-5.36)^{*}$ \\
\hline Encountered & $324(82.7)$ & $69(17.3)$ & 1 & 1 \\
\hline \multicolumn{5}{|l|}{ Disclosure status } \\
\hline No & $402(83.4)$ & $80(16.6)$ & $0.52(0.28-0.97)$ & $0.45(0.21-0.97)^{*}$ \\
\hline Yes & $125(90.6)$ & $13(9.4)$ & 1 & 1 \\
\hline \multicolumn{5}{|l|}{ Family support } \\
\hline Good & $232(89.2)$ & $28(108)$ & $1.83(1.14-2.94)$ & $2.61(1.47-4.72)^{*}$ \\
\hline Poor & $295(89.1)$ & $65(18.1)$ & 1 & 1 \\
\hline \multicolumn{5}{|l|}{ Adhernce counseling } \\
\hline Yes & 509 (85.8) & $84(14.2)$ & $3.03(1.32-6.69)$ & $2.45(0.37-1.03)$ \\
\hline No & $18(66.7)$ & $9(33.3)$ & 1 & \\
\hline \multicolumn{5}{|l|}{ Well-skilled counselor } \\
\hline Yes & $497(86.1)$ & $80(13.9)$ & $3.27(1.47-7.27)$ & $1.22(0.311-4.79)$ \\
\hline \multicolumn{5}{|c|}{ Satisfaction to counselor } \\
\hline Satisfied & $493(89.2)$ & $81(14.1)$ & $2.15(1.07-4.32)$ & $1.24(0.38-4.08)$ \\
\hline Not satisfied & 34 (73.9) & $12(26.1)$ & 1 & \\
\hline
\end{tabular}

